Case Report

\title{
Severe recurrent ascites in a Pseudo-Meigs' syndrome variant
}

\begin{abstract}
Background: Meigs' syndrome is the triad of ovarian neoplasm, ascites and pleural effusion, while Pseudo-Meigs' syndrome is the presence of ascites and pleural effusion with other pelvic tumors. Ascites resolves after tumor resection. Our case does not meet classic criteria for either but is likely a variant of Pseudo-Meigs' syndrome.

Case: A 30-year-old female had multiple Emergency Department visits for abdominal pain, distention, and shortness of breath. Imaging showed severe abdominal ascites with a large leiomyomatous uterus but no pleural effusions. Multiple paracenteses revealed benign cytology. After counselling, she underwent an abdominal myomectomy which resolved her recurrent ascites.

Conclusion: Variants of Pseudo-Meigs' syndrome should be considered in the differential of abdominal pain and leiomyomas with ascites, and surgical management should be offered.

\section{Teaching points:}

a. Variants of Pseudo-Meigs syndrome likely exist without hydrothorax

b. Though rare, Pseudo-Meigs' syndrome should be considered in the differential of abdominal pain, leiomyomas and ascites

c. If warranted, surgical management should be offered and can be curative
\end{abstract}

Volume I 2 Issue I - 202 I

\section{Ayser Al-Mshhdani MD, Claudia Payá Ten MD, Justin To MD, FACOG}

Flushing Hospital Medical Center, Flushing, NY I I355, USA

\section{Correspondence: Claudia Payá Ten, MD, Flushing Hospital Medical Center, Flushing, NY I I 355, USA, Tel (415) 740-1970, Email cpay@alumni.unav.es}

Received: January 26, 2021 | Published: February 09, 2021
Abbreviations: EKG, electrocardiogram; SLE, systemic lupus erythematosus; VEGF, vascular endothelial growth factor; FGF, fibroblast growth factor; OHSS, ovarian hyperstimulation syndrome

\section{Introduction}

Meigs' syndrome is a rare condition characterized by the presence of an ovarian neoplasm, ascites and a pleural effusion. ${ }^{1-3}$ Alternatively, Pseudo-Meigs' syndrome is characterized by the occurrence of pelvic tumors other than ovarian in origin with concurrent ascites and a pleural effusion..$^{4-5}$ Surgical resection of the tumors in both Meigs' and Pseudo-Meigs' syndrome causes resolution of the ascites and hydrothorax and therefore yields an excellent prognosis for the patient; however this diagnosis is not typically high on the differential for ascites or abdominal pain. ${ }^{6}$ Below is a presentation of a unique but likely variant case of Pseudo-Meig's syndrome with recurrent large ascites associated and uterine leiomyomas without evidence of a pleural effusion.

\section{Case presentation}

A 30-year-old nulliparous female presented to the emergency room with a chief complaint of abdominal discomfort, shortness of breath, and significant abdominal distension. On three prior occasions spanning 4 months, and prior to the Gynecology team being consulted, she was found to have severe ascites. Approximately 4 liters of benign serous fluid was drained each time via paracentesis. Cytology yielded benign pathology. She had no complaints of fevers, weight loss, or other constitutional symptoms, and had no recent travel history. She had no other significant past medical, surgical, or family history. On physical examination, she was distraught, tachypneic, and her abdominal exam showed distention, a fluid wave, and a 20 -week fibroid uterus. EKG and echocardiogram were normal. Liver function and CA-125 tests were normal, and a screen for systemic lupus erythematosus was negative. Quantiferon testing was also negative. CT scan showed a large $20 \mathrm{~cm}$ fibroid uterus and large ascites with no pleural effusion or other findings, and an MRI of the pelvis demonstrated "innumerable" uterine fibroids. It was explained to the patient that her presentation did not fit classic criteria for a specific disease, but that it could be related to her leiomyomas. After extensive counselling, she consented to an exploratory laparotomy with possible abdominal myomectomy. Intraoperatively, a complete survey showed no other abdominal pathology. 35 uterine leiomyomas were resected (largest measuring $6 \times 5 \mathrm{~cm}$ ) and 2 liters of ascitic fluid were removed. Frozen pathology demonstrated benign leiomyomas. A JP drain was placed to monitor output. The production of peritoneal fluid was minimal during her hospital stay, and she did not re-accumulate any ascites more than one year post-operatively. The final pathology showed benign leiomyomas weighing $498 \mathrm{~g}$, and the abdominal fluid cytology was benign (Figure 1A) (Figure 1B).

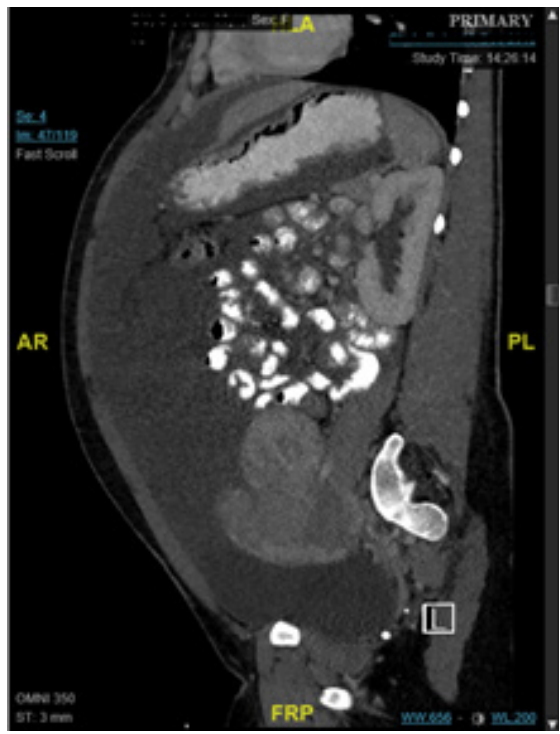

Figure IA CT scan showing large abdominal ascites. 


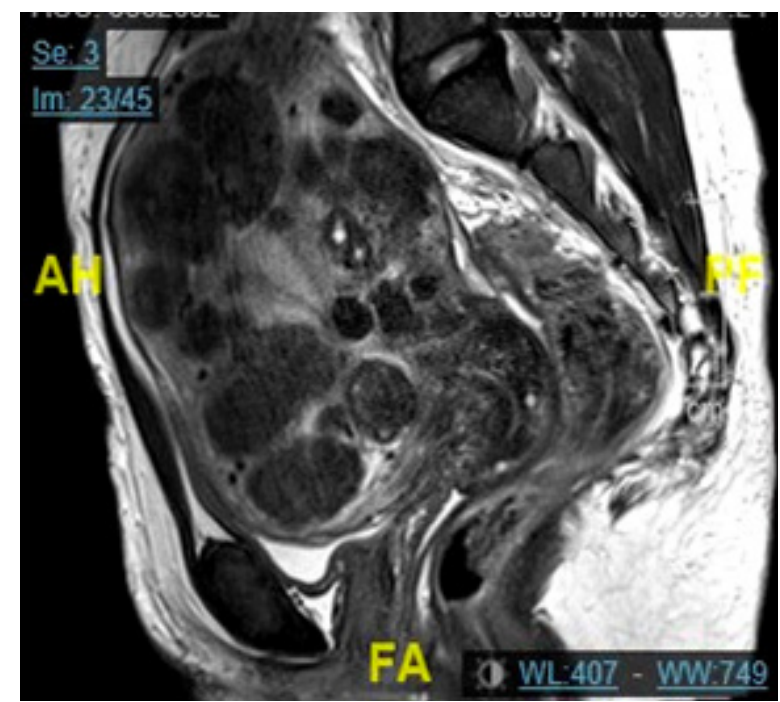

Figure IB MRI showing leiomyoma involving the entire uterus, and multiple areas of cystic degeneration.

\section{Discussion}

Due to the lack of any pleural effusion, our case did not meet the classic criteria for Meigs' or Pseudo-Meigs' syndromes. The diagnosis of Pseudo-Meigs' syndrome is associated with non-ovarian pelvic tumors, but Pseudo-Meigs' syndrome due to uterine leiomyomas is rare. ${ }^{7-11}$ Koutras et al. published the only other documented variant case of Pseudo-Meigs' syndrome without hydrothorax in an 8-yearold female who presented with bronchorrhea. ${ }^{23}$ Other more likely etiologies for ascites were evaluated for as well, including cardiac abnormalities, cirrhosis, tuberculosis, and malignancy. Related to these syndromes, Pseudo-pseudo-Meigs' syndrome (PPMS) is an extremely rare manifestation of patients with systemic lupus erythematosus (SLE), defined by the presence of ascites, pleural effusions and an elevated CA-125 level. ${ }^{12,13}$ Given that our patient's presentation most closely relates to Pseudo-Meigs' syndrome without any other more likely diagnosis, and that myomectomy led to complete resolution of the recurrent ascites, we believe that our case is a unique variant.

Our case is the first documented with such aggressive production of ascites. A total of 14 liters of fluid was drained over a 5-month period, which caused her significant morbidity. The ascites and pleural effusions in both Meigs' and Pseudo-Meigs' syndromes completely resolve after resection of the tumor. Our patient has now been ascitesfree for over 1 year. Recurrence of ascites is low, but we continue to monitor our patient given the risk for recurrent fibroids. ${ }^{8}$ The etiology of the ascitic fluid accumulation is thought to be related to lymphatic obstruction by the pelvic tumor and transudation of the interstitial edema fluid through the surface of a large tumor that exceeds the absorptive ability of the peritoneum. Another recent hypothesis of the etiology of the ascitic fluid accumulation is that cytokines, such as vascular endothelial growth factor (VEGF), fibroblast growth factor (FGF), and inflammatory cytokines play a role in the development of ascites and pleural effusions. ${ }^{14}$ In their investigation, Rizk et al. implicated VEGF as a major capillary permeability agent in ovarian hyperstimulation syndrome (OHSS). ${ }^{15}$ Similarly, VEGF has been elevated in other studies published in cases with Meigs' syndrome caused by ovarian mucinous cystadenocarcinoma and ovarian fibroma. However, these studies do not focus on uterine leiomyomas or Pseudo-Meigs syndrome and hence, factors other than VEGF must be considered. One English-language study shows the possibility of IL-6 as relevant to the pathogenesis of Pseudo-Meigs' syndrome caused by a degenerating uterine leiomyoma. ${ }^{7}$ Other potential explanations for ascites include irritation of the peritoneum by the tumor, transudation of fluid through the tumor surface in excess of the resorptive capacity of the peritoneum, hypoalbuminemia, and discrepancies between the arterial supply and the venous and lymphatic drainage. ${ }^{14}$

Regarding the procedure, this patient underwent an abdominal myomectomy only after a complete abdominal survey and frozen section confirmed benign pathology. Tuberculosis was ruled out, but malignancy is estimated to account for $7 \%$ of abdominal ascites. ${ }^{16}$ It was important to the patient to preserve her fertility as she was nulliparous. However, if the patient did not wish to conserve fertility or if the frozen pathology revealed a malignancy, it was discussed with the patient preoperatively that a total abdominal hysterectomy would be indicated. Postoperatively, we believe that follow-up should include serial exams and repeat imaging up to one year to ensure resolution of the ascites.

The CA-125 level was evaluated as it is often elevated in Meigs, Pseudo-Meigs', and Pseudo-pseudo Meigs' syndromes. It is hypothesized to be a result of the peritoneal irritation and secretion from mesothelium cells rather than a malignancy. ${ }^{17}$ Although it is widely used as a biomarker for ovarian cancer, it can also be elevated in other conditions such as peritoneal disease, benign tumors, nonovarian malignancies, and endometriosis. ${ }^{18-22}$ Our patient did not have any initial elevation, and thus we did not trend the CA-125 levels in this patient. ${ }^{23}$

\section{Conclusion}

Though rare, Pseudo-Meigs' syndrome is a disease with variants that should also be considered in the differential of abdominal pain and leiomyomas with ascites. Surgical management should be offered and may be curative.

\section{Acknowledgments}

None.

Institutional review board status: The Flushing Hospital Medical Center IRB has determined that this paper represents a case report, which does not require IRB review per the institutional guidelines. The requirement for written informed consent for the manuscript reporting clinical research on human subjects was waived by the IRB.

Précis: Variants of Pseudo-Meigs' syndrome exist which should be considered in the differential of abdominal pain and leiomyomas with ascites, and surgical management should be offered.

\section{Funding}

None.

\section{Conflicts of interest}

There is no financial support to disclose at this time. The authors whose names are listed immediately above certify that they have no affiliations with or involvement in any organization or entity with any financial interest (such as honoraria; educational grants; participation in speakers' bureaus; membership, employment, consultancies, stock ownership, or other equity interest; and expert testimony or patentlicensing arrangements), or non-financial interest (such as personal or professional relationships, affiliations, knowledge or beliefs) in the subject matter or materials discussed in this manuscript. 


\section{References}

1. McCool Dennis. Diseases of the diaphragm, chest wall, pleura, and mediastinum. Goldman-Cecil Medicine. Elsevier/Saunders; 2016.

2. Santangelo M, Battaglia M, Vescio G, et al. Meigs' syndrome: its clinical picture and treatment. Annali Italiani Di Chirurgia. 2000;71(1):115119.

3. Nemeth Alexander J, Suresh K Patel. Meigs syndrome revisited. Journal of Thoracic Imaging. 2003;18(2):100-103.

4. Liao Q, Shuhong H. Meigs' syndrome and pseudo-meigs' syndrome: report of four cases and literature reviews. Journal of Cancer Therapy. 2015;6(4):293-298.

5. Kallarackal D, Singh D. Pseudo-Meigs syndrome: A case report. Int J Case Rep Images. 2017;8(5):331-334.

6. Heilbrun ME, Olpin J, Shaaban A. Imaging of benign adnexal masses: characteristic presentations on ultrasound, computed tomography, and magnetic resonance imaging. Clinical Obstetrics and Gynecology. 2009;52(1):21-39.

7. Oguma T, Yamasaki N, Nakanishi K, et al. Pseudo-meigs syndrome associated with hydropic degenerating uterine leiomyoma: a case report. Journal of Obstetrics and Gynaecology Research. 2014;40(4):1137-1140.

8. Yaguchi A, Ban K, Koshida Y, et al. A case of pseudo-meigs syndrome caused by a giant uterine leiomyoma with cystic degeneration. Journal of Nippon Medical School. 2019;87(2):80-86.

9. Pauls M, MacKenzie H, Ramjeesingh R. Hydropic leiomyoma presenting as a rare condition of pseudo-meigs syndrome: literature review and a case of a pseudo-meigs syndrome mimicking ovarian carcinoma with elevated CA125. BMJ Case Reports. 2019;2(1).

10. Zutshi V, Tiwari S, Sirswal S. Pseudo-meigs' syndrome caused by recurrent leiomyoma uterus. Indian Journal of Gynecologic Oncology. 2018;16(4)

11. Terada S, Suzuki N, Uchida K, et al. Uterine leiomyoma associated with Ascites and hydrothorax. Gynecol Obstete Invest. 1992;33:54-58.

12. Dalvi Sr, Yildirim R, Santoriello D, et al. Pseudo-pseudo meigs' syndrome in a patient with systemic lupus erythematosus. Lupus. 2012;21(13):1463-1466.
13. Gao F, YongMei B, Yang G. Pseudo-pseudo meigs' syndrome presenting with a combination of polyserositis, elevated serum CA 125 in systemic lupus erythematosus." Medicine, 2019;98(17).

14. Ishiko O, Yoshida H, Sumi T, et al. Vascular endothelial growth factor levels in pleural and peritoneal fluid in Meigs' syndrome. Eur J Obstet Gynecol Reprd Biol. 2001;98:129-130.

15. Rizk B, Aboulghar M, Smitz J, et al. The role of vascular endothelial growth factor and interleukins in the pathogenesis of severe ovarian hyperstimulation syndrome. Hum Reprod Update. 1997;3:255-266.

16. Runyon BA, Montano AA, Akriviadis EA, et al. The serum-ascites albumin gradient is superior to the exudate-transudate concept in the differential diagnosis of ascites. Ann Intern Med. 1992;117(3):215.

17. Yadav S, Tomar S, Verma N, et al. Struma ovarii with pseudo-meigs' syndrome and raised cancer antigen-125 levels masquerading as an ovarian carcinoma: case report and literature review. Sultan Qaboos University Medical Journal. 2017.

18. Horimatsu T, Miyamoto S, Mashimo Y, et al. Pseudo-meigs syndrome caused by a krukenberg tumour of gastric cancer. Internal Medicine. 2015;54(20):2595-2597.

19. Okamoto M, Maeda K, Yanagitani A, et al. Case of pseudo-meigs syndrome caused by gastric cancer-related metastatic ovarian tumor with prolonged survival. World Journal of Gastrointestinal Oncology. 2016;8(11):80-804.

20. Yamamoto A, Miyasaka Y, Furuya K, et al. Pseudo-Meigs' syndrome due to ovarian metastases from colon cancer: a case report and review of the literature. Surgical Case Reports. 2016;2(1).

21. Yamamoto Y, Miyagawa Y, Ehara T, et al. Three cases of pseudo-meigs' syndrome secondary to ovarian metastases from colorectal cancer. Case Reports in Surgery. 2017:4604-4612.

22. Amaratunga T, Millo N, Gordon V, et al. The great mimicker: pseudomeig syndrome in a pregnant patient. a review. Ultrasound Quarterly. 2018;34(1):23-28.

23. Koutras A, Fisher S. Variant of pseudo-Meig's Syndrome. Int J Gynaecol Obstet. 1983;21(2):179-182. 